\title{
Network Synthesis and Hybrid Modeling of Miniature Electroacoustic Sensors
}

\author{
Daniel M. Warren, David E. Schafer, \\ Knowles Electronics, 1151 Maplewood Drive, Itasca, Illinois, USA, \\ daniel.warren@knowles.com
}

\begin{abstract}
:
Networks of linear, lumped parameter components are quick and efficient tools to simulate miniature microphones used in hearing aids. Synthesizing a network simulating first-order effects in a microphone is straightforward, but when subtle mechanisms such as thermal conduction at cavity walls are introduced, more advanced techniques are called for. In the first part of this paper, a potentially novel method of synthesizing a network from known solutions of the diffusion equation is introduced. In the second part, another technique is discussed in which specific physical mechanisms are not directly considered, but a model is sought which only captures the observed behavior of a microphone. Finally, the opposite end of the modeling detail spectrum is examined, and a hybrid modeling method combining detailed multiphysics finite element analysis of critical components with lumped parameter modeling of surrounding structures to leverage the best of both modeling techniques.
\end{abstract}

Key words: miniature microphone, network synthesis, lumped parameter modeling, finite element modeling, system identification

\section{Network Synthesis from First Principles}

The basic technique of developing transducer networks from first principles is well known [1] and will not be repeated here. Straightforward application of these techniques may result in unsatisfactory agreement between the model and measured data. For example, the sensitivity of a hearing aid microphone in which the front and back cavities (Fig. 1) are modeled as simple capacitors (with compliance $C_{0}=V / \gamma p_{0}$, where $V$ is the volume of the cavity, $\gamma$ is the ratio of specific heats, and $p_{0}$ is the ambient pressure) does not match measured data at low frequencies (Fig. 2).

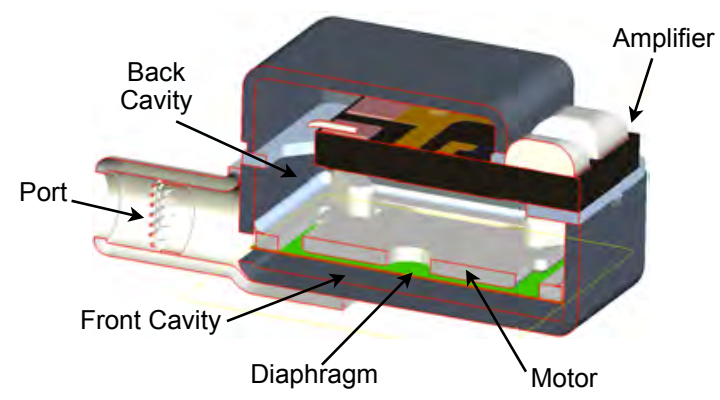

Fig 1. Cross-section view of a miniature microphone used in hearing aids.

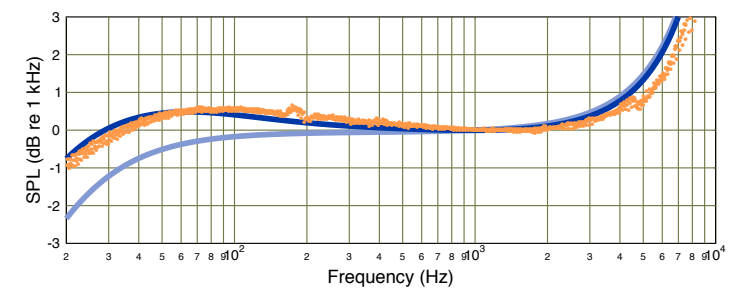

Fig. 2. Measured and predicted sensitivity of a hearing aid microphone. Measured sensitivity (orange), predicted sensitivity ignoring thermal loss (light blue), predicted sensitivity including thermal loss (dark blue).

The reason for this discrepancy is thermal conduction at the cavity walls. The walls impose an isothermal condition on an otherwise adiabatic pressure field. This effect is only evident for small cavities, as found in hearing aid microphones. Daniels [2] solves the diffusion equation with isothermal boundaries to derive the acoustical impedance of a small enclosure, which is recast here as

$Z=\left(\frac{1}{Z_{0}}+\frac{1}{Z_{T}}\right)^{-1}$

where

$$
Z_{0}=\frac{1}{j \omega C_{0}}, \quad Z_{T}=\frac{1}{j \omega C_{0}(\gamma-1) Y},
$$


and $Y$ is a function that depends upon the geometry of the cavity. For a narrow rectangular box,

$Y=\frac{\tanh \beta a}{\beta a}, \quad \beta=\sqrt{j \omega \frac{C_{p} \rho}{k}}$,

where $a$ is half the narrow dimension of the cavity and the gas constants for air $C_{p}$ is specific heat capacity at constant pressure, $\rho$ is density, and $k$ is thermal conductivity.

The fact that the acoustical impedance is written in terms of a transcendental function poses no difficulty to most calculation software. Even in Spice, a LAPLACE current-controlled voltage source could be created to simulate the impedance $Z_{T}$. However, $Z_{T}$ has a real part, which implies that it will be a source of thermal noise. It is mandatory that the network model correctly represent both the sensitivity and the self-noise of the microphone, and Spice is only able to calculate thermal noise from explicit resistors in a network.

The network synthesis method proposed here is to expand the solution of the diffusion equation as a continued fraction and derive a ladder network with that impedance [3]. The hyperbolic tangent can be expressed as a continued fraction [4]

$$
\tanh z=\frac{z}{1+\frac{z^{2}}{3+\frac{z^{2}}{5+\frac{z^{2}}{7+\ddots}}}}
$$

which, after some work, yields

$$
Z_{T}=\frac{1}{j \omega C_{T}}+\frac{1}{\frac{3}{R_{T}}+\frac{1}{\frac{5}{j \omega C_{T}}+\frac{1}{\frac{7}{R_{T}}+\ddots}}}
$$

where

$$
C_{T}=(\gamma-1) C_{0}, \quad R_{T}=\frac{a^{2} C_{p} \rho}{k(\gamma-1) C_{0}} .
$$

This impedance is recognizable as the impedance of a ladder network [5]. Truncating the ladder after 5 rungs reproduces the data much more accurately than simple compliances (Fig. 2). This same technique can be carried out for solutions of the diffusion equation in other geometries.

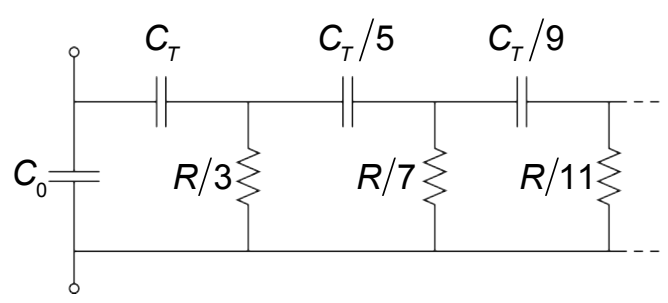

Fig. 3. Network for the compliance of a narrow rectangular cavity, synthesized from the solution of the diffusion equation.

\section{System Identification}

A very different approach to modeling microphones is not concerned with physical mechanisms, but only focuses on observed behavior. This is a method popular among control theorists, who are only interested in the behavior of a device expressed in a form that obviates important features such as stability, group delay, and controllability. The technique of system identification seeks to develop network functions characterizing the device from measured response data.

Functions that define the input and output characteristics of a network will be generally referred to as network functions $H(s)$, where $s=j \omega$ is the complex frequency in continuous time. Network functions are ratios of output to input measured across exposed ports of the network. Network functions can be expressed as ratios of polynomials

$H(s)=\frac{p(s)}{q(s)}=\frac{\sum_{k=0}^{n} a_{k} s^{n-k}}{\sum_{\ell=0}^{m} b_{\ell} s^{m-\ell}}, \quad b_{m}=1$,

with real coefficients $a_{k}$ and $b_{\ell}$ [5]. The coefficient $b_{m}=1$ avoids ambiguity among the magnitudes of the coefficients. In a black box approach, no knowledge of the device is assumed a priori. One would choose arbitrary orders $n$ and $m$ and allow a least-squares curve fitting routine search for the coefficients. The resulting functions can be faithful representations of the data, but the function tends to be unstable outside the range of the data, and the properties of the function may not mirror the properties of the microphone [6].

An alternative formulation is found by factoring the polynomials 


$$
\begin{aligned}
H(s) & =g \frac{\prod_{k=1}^{n}\left(s-z_{k}\right)}{\prod_{\ell=1}^{m}\left(s-p_{\ell}\right)} \\
& =g \frac{\left(s-z_{k r}\right) \ldots\left(\left(s-z_{k^{\prime} r}\right)^{2}+z_{k^{\prime}}^{2}\right) \ldots}{\left(p-z_{\ell r}\right) \ldots\left(\left(p-z_{\ell^{\prime} r}\right)^{2}+p_{\ell^{\prime}}^{2}\right) \ldots} .
\end{aligned}
$$

The roots $z_{k}$ of $p(s)=0$ are the zeros of the function and the roots $p_{\ell}$ of $q(s)=0$ are the poles. Since the coefficients in Eq. (1) are real, the zeros must either be real valued $z_{k}=z_{k r}$ or occur in conjugate pairs $z_{k^{\prime}}=z_{k^{\prime} r} \pm j z_{k^{\prime} i}$, as do the poles. The product of the conjugate pair factors are written in the form $\left(\left(s-z_{k^{\prime} r}\right)^{2}+z_{k^{\prime}}^{2}\right)$, which is a convenient form for calculation as it eliminates small spurious imaginary factors resulting from the numerical product of $\left(s-z_{k}\right)\left(s-z_{k}^{*}\right)$.

In general, network functions are complex in value, and it is best to work with complex data when available. In the absence of complex data, fitting the function

$$
20 \log _{10}\left|H\left(g, z_{1}, z_{2}, \ldots, p_{1}, p_{2}, \ldots ; s\right)\right|
$$

to the data expressed in decibels is another option. This has the advantage of being capable of recovering the phase of the data values, assuming the system is minimum phase [7].

A practical consideration when fitting curves is scaling. If the free parameters in a curve fit have widely disparate orders of magnitude, than the gradient matrix is ill-conditioned and the smaller parameters may get lost in the round-off. At the very least, the smaller parameters are not determined to the same precision as the larger parameters, if they are found at all.

First, we scale the frequency. Choose the maximum frequency of interest $f_{\max }$. It is advisable that this frequency be greater than the highest frequency in the data. In fact, if the eventual goal is to create a discrete-time filter to represent the data, the Nyquist Theorem requires that $f_{\max }$ be at least twice the highest frequency in the data. Next, choose a value $v_{\text {mid }}$ somewhere in the middle of the range. Normalize each data set as
$\left.\left\{\left\{\Omega_{i}, \bar{v}_{i}\right\}, \ldots\right\}=\left\{\left\{\frac{\pi f_{i}}{f_{\text {max }}}, \frac{v_{i}}{v_{\text {mid }}}\right\}\right\}, \ldots\right\}$,

where $0 \leq \Omega \leq \pi$ is the normalized circular frequency.

Although the network function is complex valued, it is required that the free parameters and the independent variable of the network function are real. Complex parameters are broken into their real and imaginary parts as two separate parameters as in Eq. (2). Eq. (2) is a function of complex frequency $s$, so for the curve fit we set $s=j \Omega$, and fit the function to the normalized data. Finally, we express the fit function in the original scale by mapping $\Omega$ back to the complex frequency $s$ and multiplying by the magnitude of the original data set

$\left|v_{\text {mid }}\right| H(j \Omega): j \Omega \rightarrow \frac{s}{2 f_{\max }}$.

In order to have control over the fitting process, a software tool was developed in Mathematica, shown in Fig. (4). The tool allows the user to interactively place poles and zeros in the third quadrant of the $s$-plane. Poles or zeros placed on the origin are locked to the origin, and those placed on the negative real axis are locked to, but free to vary along, the real axis. Poles and zeros placed elsewhere represent complex conjugate pairs and are free to vary within the constrained plane

$$
\{s: \Re(s)<0 \wedge 0<\mathfrak{I}(s) \leq \pi\} .
$$

The tool dynamically creates a function of the form Eq. (2) and displays its Bode plot with the normalized data. Once the user is satisfied that the function has the right structure and reasonable initial conditions, he can push a button and the curve fit routine finishes the job. 


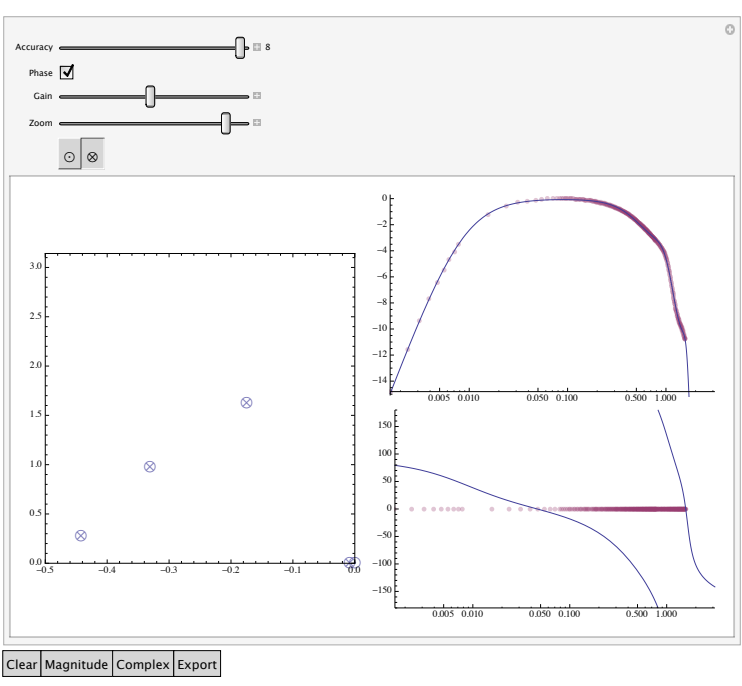

Fig. 4. A screen from the system identification tool, shown fitting the transfer function of a microphone sensitivity (right) to a pole/zero model (left). Only magnitude data was available, and the phase Bode plot shows the reconstructed phase.

This tool automatically constrains the function to be stable and minimum-phase, which are expected properties of a transducer network. Constraints chosen by the user are adhered to during the curve fit. The tool is designed to allow the user to apply prior knowledge of the problem domain to system identification.

The network functions derived using this tool are more likely to have the same properties as the device than a black box system identification because the user is able to input expected behavior into the fitting process. However, the data the modeler has to work with is necessarily band-limited, and out-of-band features, which are only partially captured in the data, are, to a certain extent, ambiguous. For example, it is already known that the correct model for the microphone response in Fig. 4 should have first-order high-pass, two secondorder low-pass, and a first-order low-pass characteristics. Since the first-order low-pass is close to the high frequency limit of the data, and one second-order low-pass peaks is beyond it, the curve fit cannot resolve these features correctly, finding a third second-order low-pass peak instead.

At this level, then, system identification can only be considered a model of the data, not a model of the system. For this reason, the author has not seriously pursued the possibility of using Foster or Cauer synthesis to develop a network solely from network functions determined this way. The resulting network would not necessarily represent the mechanisms in the microphone, and having a network that just represents the data is not a significant improvement over having a continuous-time function that does the same thing. It seems more fruitful to derive the network functions from a network constructed by conventional means, then fit those functions to measured data.

\section{Hybrid Modeling}

Now we move to a modeling style where we need more detail, not less. Lumped parameter modeling is simple, fast, and convenient because it glosses over the details. Many parameters in lumped models are "effective" values, meaning they are somewhat modified from the value one would predict from first principles based on a simpler geometry which is more amenable to analysis than the actual shape of the part.

At the other end of the spectrum is Finite Element Analysis (FEA), which, despite impressive development in software, remains anything but simple, fast, and convenient. It is, however, the best means of analyzing complex geometries and integrating conflicting requirements among several physical domains. Also, FEA encourages beginning users to simulate a single system geometry to derive a single result, which provides no insight into the nature of the system. In other words, it is easy to get an answer from FEA, but to get enlightenment requires additional effort.

Understanding a system using FEA requires multiple simulations of similar systems, perturbed from the original in controlled ways. This is difficult to do when a single FEA solution of an entire multiphysics system in three dimensions can take hours to run and take more memory than available in a practical computing system. So, how do we leverage the power of FEA within the time constraints of a product development cycle?

The approach we chose to take (described in more detail in [8]) was to segment the microphone into easy and hard parts. Much of a miniature microphone can already be simulated using lumped parameter models as previously discussed. Aspects of the design which we do not intend to modify, such as the back volume, front volume, and geometry of the port and outlet tube, can be calculated in isolation in one-time FEA simulations and captured in a lumped parameter circuit (Fig. 5). 
-

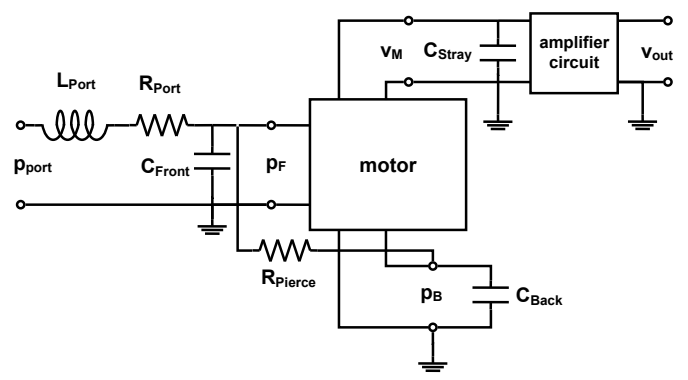

Fig. 5. Lumped parameter network showing the simpler and immutable components in the design optimization.

Our task was to optimize the design of the working part of the microphone, the motor (Fig. 6), so it is modeled in exacting detail in FEA. The motor consists of a tensioned diaphragm closely spaced to a metal backplate coated with an electret material maintaining a permanent electric field within the narrow gap.

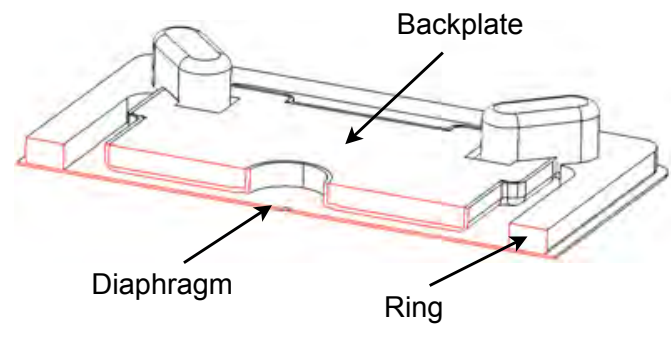

Fig. 6. Cross-section of a microphone motor.

Our primary figure of merit is the signal to noise ratio (SNR) of the microphone. The signal is the sensitivity of the microphone to an acoustic pressure, which relates primarily to the surface voltage on the electret, the gap spacing, and the tension of the diaphragm, but is peripherally (negatively) effected by parallel capacitive paths from the high-potential backplate to ground through nearby conductive surfaces (the amplifier circuit, diaphragm tension ring, and the metallic can encasing the microphone). The noise contribution from the motor is all about air motion. As noted previously, any dissipative mechanisms in the system exhibit as noise. The narrow gap between the diaphragm and backplate is a squeezed film of air and can be very resistive to air flow. Small holes and peripheral notches are provided in the backplate to relieve dynamic pressure developed due to motion of the diaphragm, but the noise of the is highly dependent upon the distance the air has to travel to get through the reliefs. On the converse, any metal we remove from the backplate cannot develop electrical signal to sense the diaphragm motion, lowering sensitivity.
Optimizing the motor design requires the careful simultaneous optimization of electrostatic, electrodynamic, mechanical, and boundary layer acoustical factors. An FEA simulation was set up to include the motor and conductive surface representing the cup and circuit conductors (Fig. 7). Critical parameters, including dimensions controlling the shape of the backplate perimeter and a central hole, the gap spacing, the equivalent voltage on the electret, and diaphragm tension are variables in the optimization problem. The remainder of the system, such as acoustic volumes and acoustic porting, which are considered constants in the problems, are embodied as a circuit (Fig. 5), to which the FEA results are coupled.

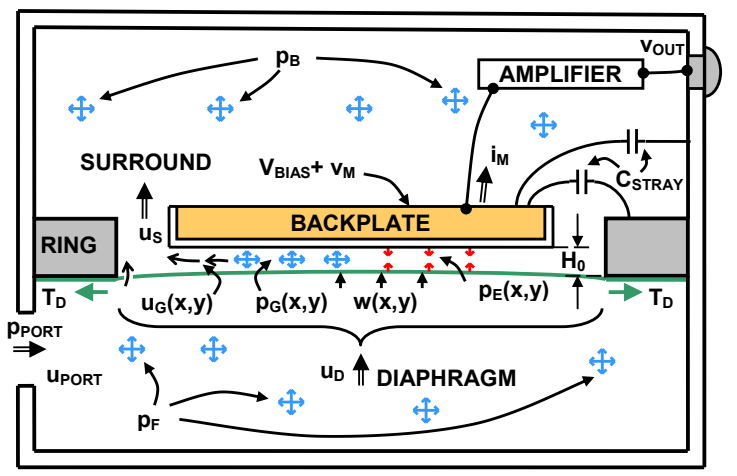

Fig. 7. Diagram of interacting fields and quantities in within a microphone.

Several pieces of software have to work together. A master program was developed in Mathematica. The master program contains a list of the current and previous parameter values. The geometry and boundary conditions for the FEA are developed by the master program and checked for consistency, then written to a COMSOL control file. COMSOL runs, writing the results to an output file. The master program reads the output file and reduces the results to admittance relations among the front and rear acoustic pressure and volume velocity, and voltage and current from the backplate. Finally, the network is evaluated for sensitivity and noise.

This work started in an older version of COMSOL, when it was called FEMLAB. In a future project we will convert the software to a recent version of COMSOL, and see if the Acoustics, AC/DC, and Optimization modules can solve this problem entirely within COMSOL.

The process is repeated, modifying the critical parameters to follow the steepest descent contour, until the sensitivity to noise ratio is maximized. Tab. 1 illustrates the results of one optimization, yielding a $2 \mathrm{~dB}$ improvement in signal to noise (on an A-weighted scale.) The specific dimensions are not shown as they 
relate to an unreleased product and are considered proprietary.

Tab. 1. Tabulated results from an optimization of a microphone motor, showing changes in parameter values relative to starting values, and resulting improvement in signal to noise ratio.

\begin{tabular}{lc}
\hline \multicolumn{1}{c}{ Parameter } & $\begin{array}{c}\text { Change After } \\
\text { 206 Cases }\end{array}$ \\
\hline Air Gap & $-21 \%$ \\
Diaphragm Tension & $+12 \%$ \\
Electret Bias Voltage & $+30 \%$ \\
Dimension "A" & $+65 \%$ \\
Dimension "B" & $-38 \%$ \\
Dimension "C" & $-84 \%$ \\
Dimension "D" & $+32 \%$ \\
Dimension "E" & $+65 \%$ \\
Input-Referred Noise & $-1.93 \mathrm{dBA}$ \\
\hline
\end{tabular}

\section{Conclusions}

Three methods for modeling microphones at varying levels of detail and utility have been presented, through mathematical analysis of governing equations, numerical analysis of measured data, or mixed finite element and lumped parameter analysis. Each method has applicability depending upon the level of accuracy required in the solution or the level of system knowledge the modeler has access to.

\section{References}

[1] L. Beranek, Acoustics, Acoustical Society of America, New York, (1993); ISBN: 0-88318-494$\mathrm{X}$.

[2] F. B. Daniels, "Acoustical Impedance of Enclosures". The Journal of the Acoustical Society of America, 19(4), 569-571 (1947); doi:10.1121/1.1916522.

[3] D. M. Warren and J. L. LoPresti, "A ladder network impedance model for lossy wave phenomena". The Journal of the Acoustical Society of America, 119, 3377 (2006).

[4] NIST Digital Library of Mathematical Functions. http://dlmf.nist.gov/4.49.E1, Release 1.0 .5 of 2012-10-01.

[5] M. E. van Valkenburg, Introduction to Modern Network Synthesis. Wiley, New York, (1960); ISBN: 0-471-89991-7.

[6] D. M. Warren, "Applications of network synthesis and zero-pole analysis in transducer modeling". Proceedings of Meetings on Acoustics, (to be published after ASA/ICA 2013).
[7] A. V. Oppenheim and R. W. Schafer, DiscreteTime Signal Processing, Third Edition, Pearson, New Jersey, (2010); ISBN: 0-13-198842-5.

[8] D. E. Schafer, "Electret microphone modeling and optimization by combined finite element analysis (FEA) and lumped-element techniques". Proceedings of Meetings on Acoustics, (to be published after ASA/ICA 2013). 